\title{
Bragg Reflection Transmission Filters \\ for Variable Resolution Monochromators
}

\author{
Dean Chapman \\ National Synchrotron Light Source \\ Brookhaven National Laboratory \\ Upton, New York 11973
}

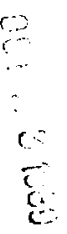

\begin{abstract}
There are various methods for improving the angular and spectral resolution of monochromator and analyzer systems. The novel system described here, though limited to higher $x$-ray energies ( $>20 \mathrm{keV}$ ), is based on a dynamical effect occurring on the transmitted beam with a thin perfect crystal plate set in the Bragg refiection case.
\end{abstract}

In the case of Bragg reflection from a zerfect crystal, the incident beam is rapidly attenuated as it penetrates the crystal in the range of reflection. This extinction length is of the order of microns. The attenuation length, which determines the amount of normal transmission through the plate is generally much longer. Thus, in the range of the Bragg reflection the attenuation of the transmitted beam can change by several orders of magnitude with a small change in energy or angle.

This thin crystal plate cuts a notch in the transmitted beam with a width equal to its Darwin width, thus acting as a transmission filter. When used in a non-dispersive mode with other morochromator crystals, the filter when set at the Bragg angle will reflect the entire Darwin width of the incident beam and transmit the wings of the incident beam distribution. When the element is offset in angle by some fraction of the Darwin width, the filter becomes useful in adjusting the angular width of the transmitted beam and removing a wing. Used in pairs with a symmetric offset, the filters can be used to continuously adjust the intrinsic angular divergence of the beam with good wing reduction. 
Instances where such filless may be useful are in improving the angular resolution of a small angle scattering camera. These filters may be added to a Bonse-Hart camera with one pair on the incident beam to reduce the intrinsic beam divergence and a second pair on the analyzer arm to improve the analyzer resolution. Also these filters could be used in dispersive monochromator systems to improve the energy resolution.

*Contact author for proofs: Dean Chapman

National Synchrotron Light Source

Brookhaven National Laboratory

Upton, New York 11973 


\section{Principles of Operation}

In the case of Bragg reflection for a perfect crystal, the incident beam is attenuated rapidly as it penetrates the crystal in the range of reflection (secondary extinction). Typically, the extinction length is in the order of microns for a perfect crystal when the diffraction condition is met. For example, the extinction length for a silicon (111) reflection at $20 \mathrm{keV}$ is 15 microns. In contrast the length over which normal attenuation (photoelectric plus scattering) occurs is generally much longer. Again for silicon at $20 \mathrm{keV}$, the attenuation length is 1.1 millimeters. Thus, the transmission of a monochromatic $\mathrm{x}$ ray beam through a thin perfect crystal plate can change by several orders of magnitude with a small change in energy or angle. A symmetrically silicon (111) plate, $0.05 \mathrm{~mm}$ thick, has a 5.67 degree Bragg angle and an effective thickness of $.506 \mathrm{~mm}$ at $20 \mathrm{keV} \mathrm{x}$-ray energy. The transmission through such a crystal away from the diffraction condition is about 0.63 ( $1 / 2$ attenuation length ). The transmission at the Bragg angle is about $2 \times 10^{-15}$ ( 33 extinction lengths! ).

A plot of a crystal's transmitted and reflected beans, as described above, is shown in figure 1. The figure shows the rocking curve of the reflected beam (dotted line) for comparison with the transmitted beam (solid line) and shows the transmission as a function of rocking angle. Note the sharp onsat of attenuation as the crystal enters the near total reflection range. The calculated change in transmission is about 14 orders of magnitude with an angular change of 0.3 arc-seconds! The above calculation and subsequent calculations were done with the MONO program ${ }^{[1]}$. This progratn is based on Zachariasen's development of the dynamical theory of diffraction ${ }^{[2]}$.

This thin crystal plate cuts a notch in the transmitted beam with an angular wideh equal to its Darwin width, therefore, acting as a transmission filter. By using the same crystal type and reflection for the filler as is used for the monochromator, the crystal, when set at the Bragg angle will reflect the entire Darwin width of the incident beam and transmit the wings. When the element is offset in angle by some fraction of the Darwin width, the filter becomes useful in adjusting the angular width of the transmitted beam and removing a wing. Used in pairs with a symmetric angular offset, the filters can be used to continuously adjust the intrinsic angular divergence of the beam with good wing reduction. 
The throughput of a filter, as described above, improves with increasing $x$-ray energy. This can be easily understood by looking at the energy dependence of the extinction length, absorption length and effective crystal thickness. For a fixed thickness crystal plate, the effective thickness at or near the Bragg angle, increases linearly with $x$-ray energy. In the absence of absorption edges, the extinction length increases linearly with energy. Thus, the effective number of extinction lengths of a fixed thickness crystal plate, as a function of energy, is fixed. However, the absorption length increases as approximately the cube of the $x$-ray energy in the absence of absorption edges, ignoring the attenuation due to coherent and Compton scattering. The effective number of absorption lengths the fixed thickness plate presents decreases as the inverse square on the $x$-ray energy. The effective number of extinction lengths determines the attenuation for rays within the Darwin width of the crystal while the effective number of attenuation lengths determines the attenuation for rays outside the Darwin width of the crystal ( normal transmission).

For a practical transmission filter, the crystal plate must be thin enough so that outside of the Danwin width, the normal transmission is high ( $>50 \%$ ). However, the plate cannot be fabricated arbitrarily thin and also be mechanically stable. For silicon, a reasonable lower limit on crystal thickness is approximately 50 microns. Silicon (111) filters becomes a usable element to improve the instrument resolution near and above $20 \mathrm{keV}$.

\section{Examples of Applications}

When the dispersion of all the monochromator or analyzer elements match, including the transmission filters, the effect of beam divergence will not change the performance of the filter. Figure 2 shows, the effect of two 50 micron transmission flters used to reject the wings of the throughput of a silicon (111) double crystal monochromator set at 50keV. The plots show the logarithm of the expected throughput as a function of vertical beam divergence ( 0.05 milliradians ) and relative $x$-ray energy. Figure $2 a$ shows the throughput without transmission filters and figure $2 \mathrm{~b}$ shows the addition of two filters set at plus and minus one are-second relative to the Bragg angle. At this energy, one arc-second corresponds to the Darwin width of the Si (111) reflection. Note the rejection of the monochromator wings in the 
range of the Darwin width of the filters.

When such a monochromator arrangement is used with a similar analyzer (double crystal Si (111) reflection plus two $\mathrm{Si}$ (111) transmission filters ), then a small angle camera can be made with sub-arcsecond resolution at 50keV. The background normally encountered at very small angles would be almost completely eliminated with such a system due to the very strong wing reduction provided by the filters.

A final example of the use of the silters to provide a high energy resolution beam using a dispersive crystal arrangement. The monochromating system is a four crystal dispersive geometry. The first and last pair of crystals are parallel with the second and third crystal set in a dispersive geometry. The normalized throughput of this arrangement is shown in figure 3a. All crystals are Si (111) with a average $\mathrm{x}$-ray energy of $20 \mathrm{keV}$ and 0.1 milliradians of vertical divergence in the incident beam. Figure $3 \mathrm{~b}$ shows the addition of two pairs of transmission filters each 50 microns thick also in a dispersive geometry to reduce the wings and improve the energy resolution. Each of the filters is offset symmetrically from the Bragg angle by 2.5 arc-seconds for both the dispersive and non-dispersive sets. Note that the peak throughput has been reduced by an order of magnitude, however, the FWHM energy spread has been reduced from about $3 \mathrm{eV}$ to $1.5 \mathrm{eV}$ at $20 \mathrm{keV}$ and the wings have been suppressed several orders of magnitude.

\section{Conclusion}

The use of transmission filters may provide a relatively simple method for the design of high energy variable resolution monochromators. Since the filters work in a transmission mode they can be added to a working system with liule redesign.

Currently, plans are under way to fabricate and test the operation of such a device at the NSLS with the first application the development of a high energy small angle scattering camera on the X17 Superconducting Wiggjer Beamline. 
$-6$.

This work supported by the United States Department of Energy under contract \#DE-AC02-76CH00016. 


\section{Figures}

Figure 1 The Calculated Transmission (Solid Line) and Reflection (Dotted Line) Rocking Curve of a 50 micron thick Symmetric Bragg Case Silicon (111) Plate at 20keV.

Figure 2 Calculated Double Crystal Si (111) Monochromator Throughput with 0.1 milliradian of Beam Divergence in the Diffraction Plane at an Average $X$-Ray Energy of 50keV. (A) Without Transmission Filters. (B) With Two 50 micron Si (111) Filters Symmetrically Offset from the Bragg Angle by 1 arc-second. Logarithmic Throughput Axis.

Figure 3 Calculated Four Crystal Dispersive Si (111) Monochromator Throughput with 0.1 milliradian of Beam Divergence at $20 \mathrm{keV}$ X-Ray Energy. (A) Without Transmission Filters. Note the X Pantem Arising from the Crossing of each Crystal Pair's Dispersion. (B) With Four 50 micron Si (111) Filters Symmetrically Offset from the Bragg Angle by 2.5 arc-seconds for both the Dispersive and Non-Dispersive Crystal Pair. Logarithmic Throughput Axis. 


\section{References}

[1] D. Chapman, MONO: A Program to Calculate Monochromator Performance for Synchrotron Beamlines", presented at SRI, 1989, Lawrence Berkley Laboratory, published elsewhere in these preceedings.

[2] W. H. Zachariasen, "Theory of X-Ray Diffraction in Crystals", John Wiley and Sons, Inc., New York, (1945). 
Normalized Throughput

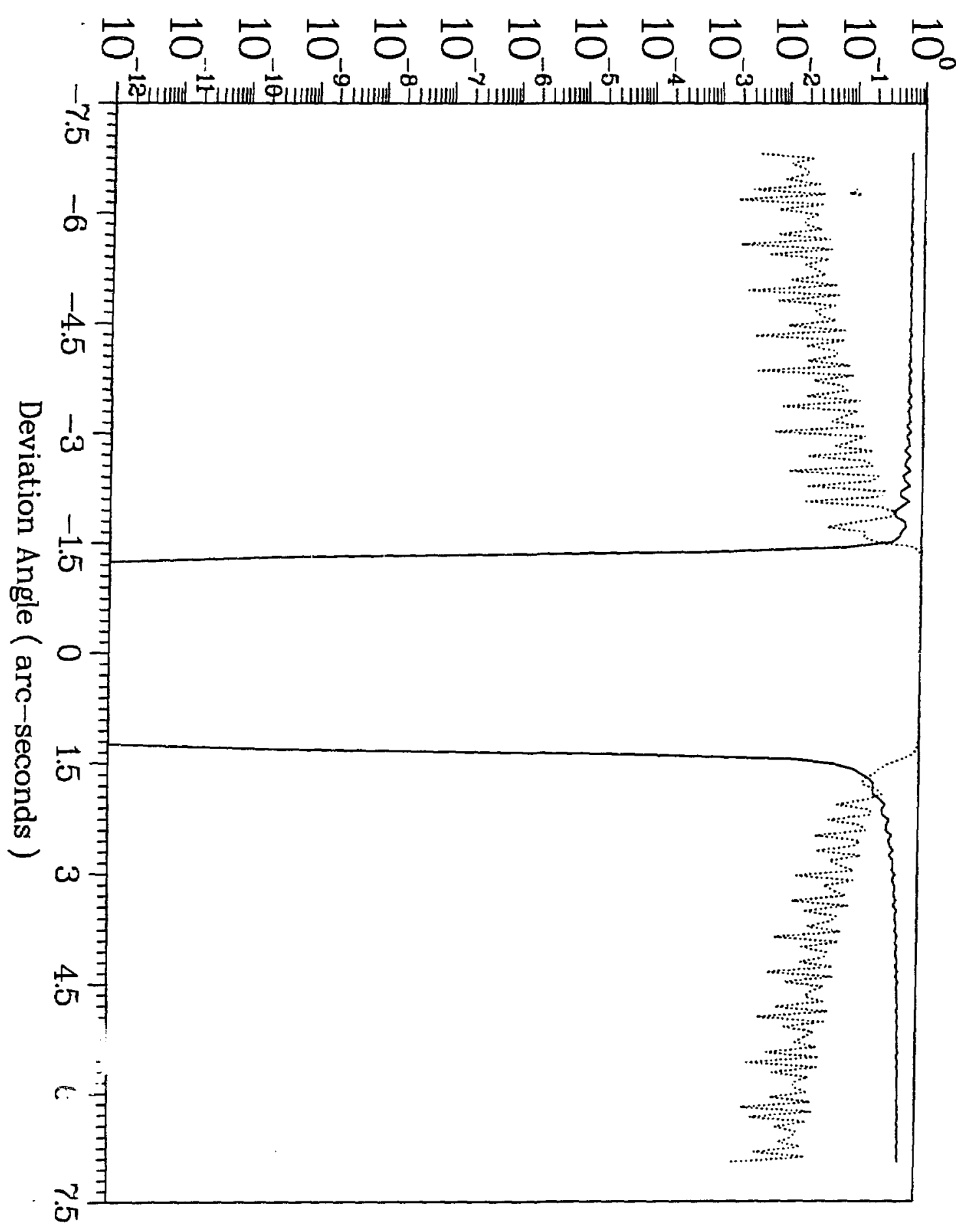




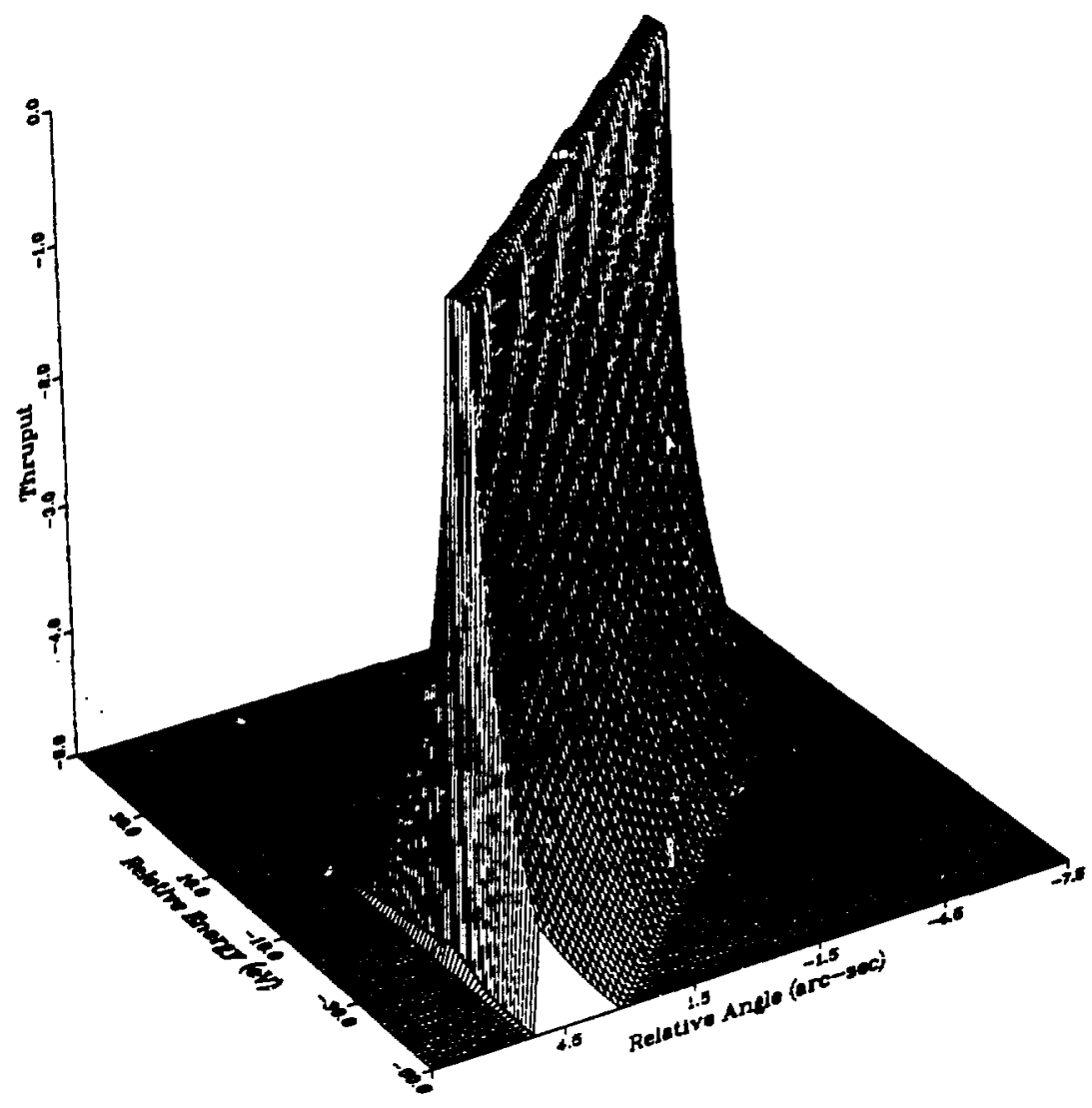




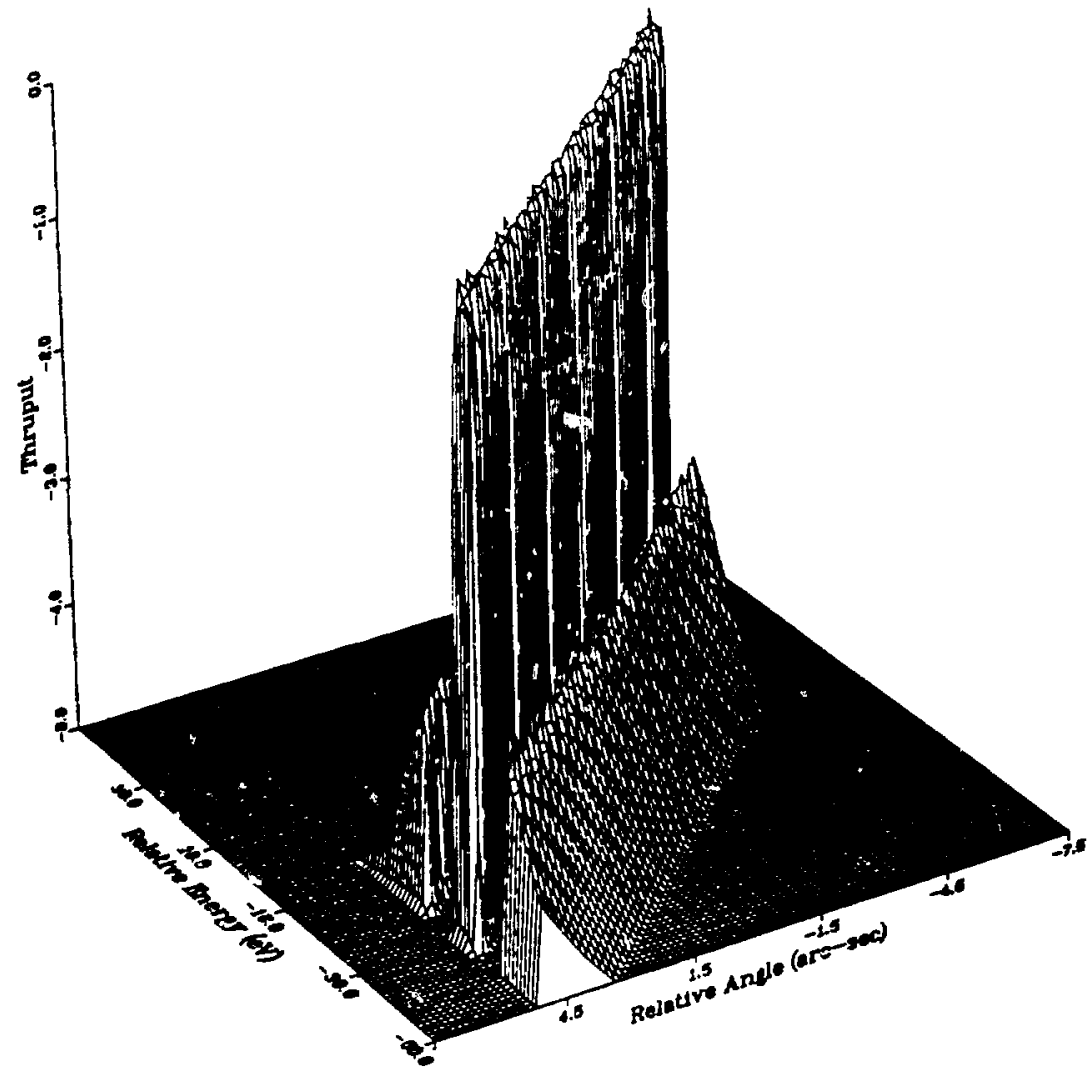




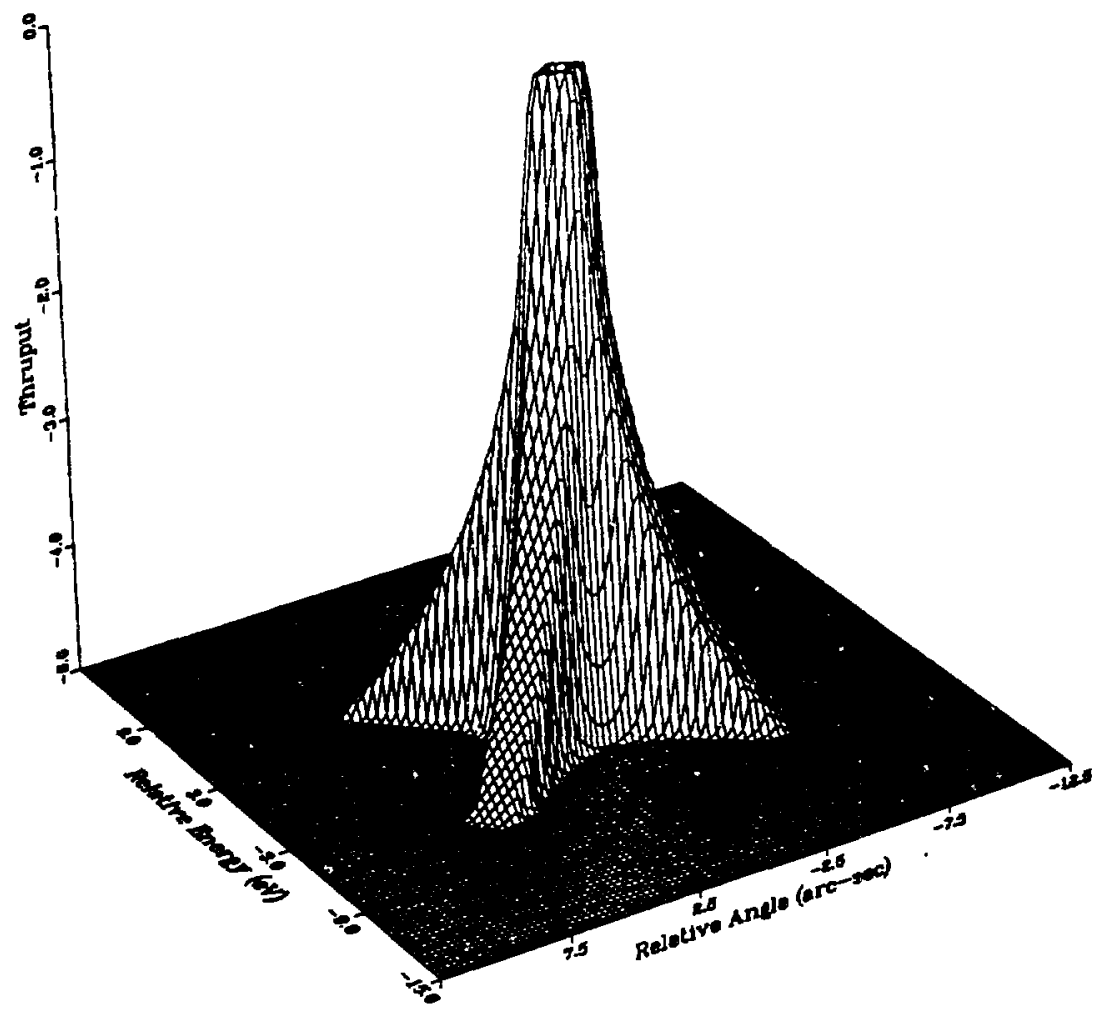




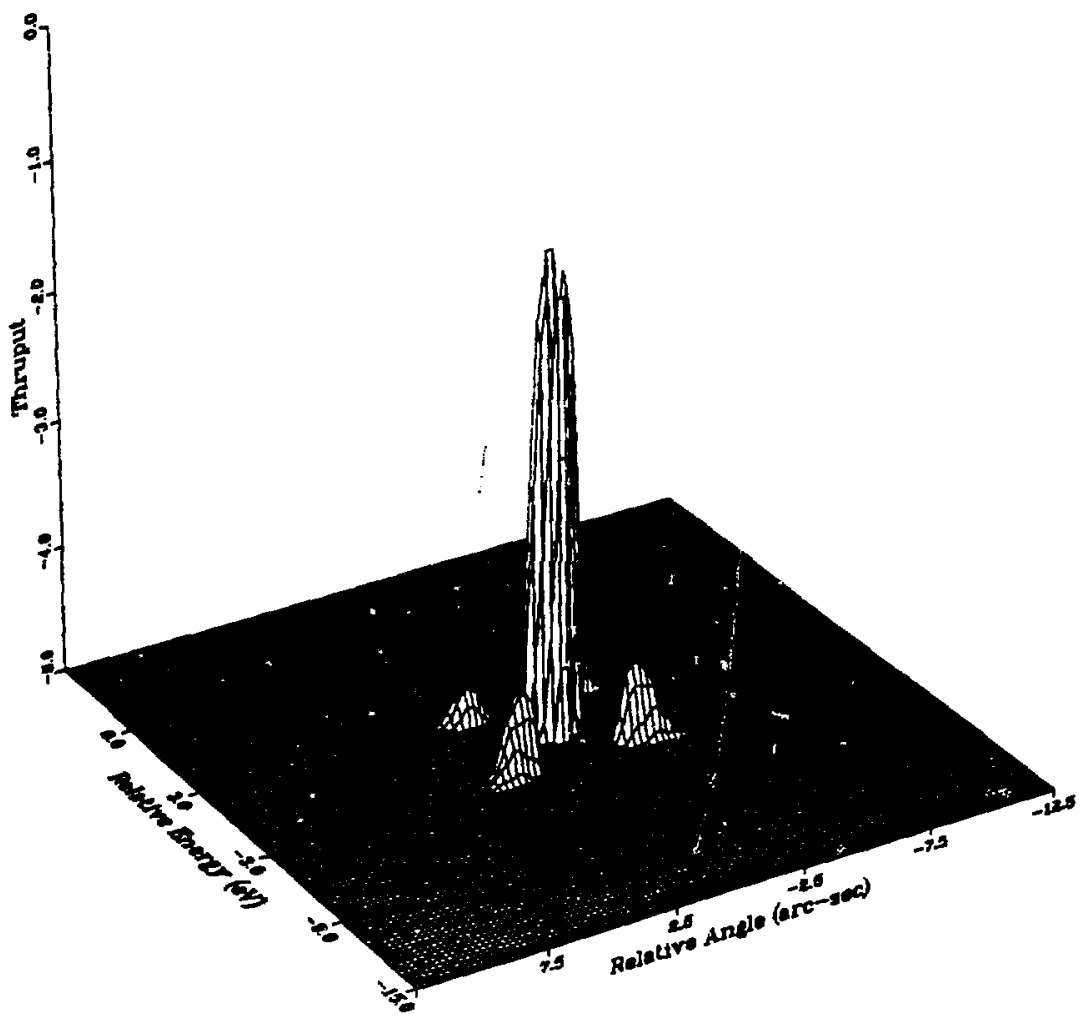

